\title{
Efectos del ejercicio aeróbico sobre los estados de ánimo en mujeres mayores Effects of aerobic exercise on the moods in elderly women
}

\author{
Pedro Jesús Ruiz Montero*, Antonio Baena Extremera** \\ *Universidad de Málaga, **Universidad de Murcia
}

\begin{abstract}
Resumen: El propósito de este estudio descriptivo es evaluar el grado de influencia que la práctica de actividad física aeróbica ejerce sobre el bienestar psicológico, la salud mental y su repercusión en los estados de ánimo. Se ha utilizado una muestra formada por 63 mujeres participantes del programa de Actividad Física para mayores de la provincia de Málaga, con edades comprendidas entre los 60 y 79 años y que pertenecen a tres poblaciones inferiores a 3.000 habitantes, en una comarca denominada «Sierra de las Nieves». Las participantes cumplimentaron un cuestionario sociodemográfico estandarizado y el Profile of Moods States, POMS, en una versión reducida de 30 ítems agrupados en cuatro factores: tensión (8 ítems), vigor (8 ítems), fatiga ( 7 ítems) y amistad ( 7 ítems). Los resultados obtenidos muestran que los cuatro estados de ánimo evaluados no están asociados con las variables edad y motivos de práctica física, mientras que si está asociada la antiguiedad de práctica de actividad física con el vigor y la variable enfermedad con la tensión.
\end{abstract}

Palabra clave: Actividad física; ánimo; salud mental; personas mayores; mujer.

Abstract: The intention of this descriptive study is to evaluate the degree of influence that the practice of aerobic physical activity exerts on the psychological well-being, the mental health and its repercussion in the moods. A sample formed by 63 participant women of the program of Physical Activity for elderly people has been used of the province of Malaga, with ages between the 60 and 79 years and that belongs to three inferior populations to 3,000 inhabitants, in a denominated region «Sierra de las Nieves». The participants complimented a sociodemografic questionnaire standardized and Profile of Moods States, POMS, in group a reduced version of 30 items in four factors: tension ( 8 items), vigor ( 8 ítems), fatigue ( 7 ítems) and friendship ( 7 items). The results obtained show that the four evaluated moods are not associate with the variables age and practice for physical reasons, whereas if the antiquity of practice of physical activity with the vigor and the variable disease is associate with the tension.

Key words: Physical activity; mood; mental health; elderly people; woman.

\section{Introducción}

Desde los orígenes del ser humano, éste se ha preocupado por mantenerle el pulso a la naturaleza (Moreno, 2005), con vista de conseguir una vida sana y con ello, una óptima salud integral. Se ha adaptado a la evolución y cambios en la historia, alcanzando de esa manera el bienestar y la calidad de vida. Para conseguir esta última y tener una vida saludable, ha sido necesario tener en cuenta desde los efectos de las personas sobre la salud (Acuña y Bruner, 2002), hasta llegar a la salud de las personas mayores desde diferentes ámbitos, como puede ser la nutrición y alimentación, bienestar físico o factores psicológicos entre otros. Los beneficios que la actividad física provoca en la salud (Márquez, 1995; Endres et al, 2003; Lee, Folsom y Blair, 2003), fundamentan un hecho que se manifiesta cada vez más latente y que es planteado desde una perspectiva multidisciplinar, no marcándose como único fin la prolongación de la vida, sino que buscan el mantenimiento y mejora de calidad de esta. En el lado opuesto, los perjuicios que conlleva el sedentarismo han sido recientemente revisados, poniendo de manifiesto la relación inversa que existe entre el nivel de actividad física y el riesgo de sufrir enfermedades mentales o estados de ansiedad, y depresión (Varo, Martínez, y Martínez-González, 2003). Resulta por tanto de vital importancia, focalizar la investigación en promover un envejecimiento saludable más que limitarse a tratar las diversas enfermedades que se asocian a la vejez (Abbott, 2004).

La actividad física parece ser uno de los factores que más claramente ayuda a la longevidad (Dorn, Naughton, Imamura, y Trevisan, 1999; Erkssen, 2001), mediante el aumento del ejercicio variado (Myers, et al., 2002; Gulati et al., 2003) y la adaptación a las capacidades actuales de las propias personas (Rosenfeld y Tenembaum, 1992).

En la Segunda Asamblea Mundial sobre el Envejecimiento de las Naciones Unidas (Madrid, 2002) se reconoce que de aquí a 2050, el número de personas con más de 60 años aumentará de 600 millones a casi 2.000 millones. Se pasará de un $10 \%$ a un $21 \%$, experimentándose el mayor incremento en los países desarrollados. En cuanto a mujeres,

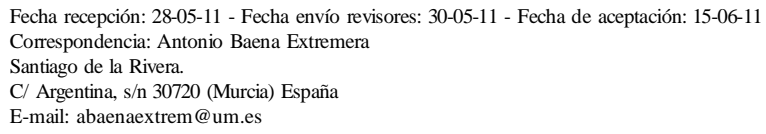

Fecha recepción: 28-05-11 - Fecha envío revisores: 30-05-11 - Fecha de aceptación: 15-06-11

E-mail: abaenaextrem @um.es

su valor medio será mayor que el de los hombres (100 mujeres frente a 78 hombres).

Esta población tan específica como son las personas mayores y concretamente las mujeres, tienen una característica fundamental, los hábitos negativos adquiridos a lo largo de toda su vida (Dannery Edwards, 1992). Por ello, se debe tener especial atención al planificar y realizar programas de actividades físicas para personas mayores. La importancia de esto último se hace más significativa cuando se ha constatado que si se práctica actividad física y deportiva con asiduidad y respetando una frecuencia constante, se retrasa a la vez que reduce una futura incapacitación (Daniels, Van Rossum, De Witte, Kempen, y Van den Heuvel, 2008). Concretamente en nuestro país, se realizan unos estudios periódicos que consisten en el análisis del vínculo entre ciudadanos y deporte (García Ferrando, 2005), llegando a la conclusión de que la práctica física y deportiva va disminuyendo a la vez que se avanza en edad.

Sireducimos la práctica de ejercicio físico a programas de actividades aeróbicas y anaeróbicas, diferentes autores (McCann y Colmes, 1984) coinciden en los beneficios que se producen sobre la salud mental de los individuos (Morales y Coto, 2004). En un estudio realizado con mujeres mayores, se demostró que las actividades físicas aeróbicas producían cambios significativos en los estados de ánimo, incluso veinticuatro horas después de su realización (Maroulakis y Zervas, 1993). Dishman (1994), destaca igualmente el interés que el ejercicio tiene en los aspectos psicológicos, sobre todo aquellos relacionados con los cambios en los estados de ánimo y con el nivel de satisfacción de una actividad física regular. Sobre esto, Olmedilla, Ortega y Madrid (2008) afirman que la relación entre factores psicológicos y práctica de actividad física parece tener un sentido de reciprocidad, más que de unidireccionalidad; es posible que la práctica de ejercicio físico beneficie a la salud psicológica, pero también ésta puede hacer más viable la posibilidad de practicar algún tipo de ejercicio.

Otros estudios muestran que la práctica de ejercicio físico condiciona la calidad de vida de forma positiva (Biddle, 1993), a la vez que el estado físico y psicológico (Benaziza, 1998, Jiménez, Martínez, Miro y Sánchez, 2008), mejorando la emotividad y estado de ánimo (Biddle, Fox y Boutcher, 2000) e incrementando la autoestima (McAuley, Mihalko y Bane, 1997). No importa el periodo de práctica física, pudiendo ser más largo o corto. Ambos van a garantizar beneficios en el bienestar psicológico (Dishman, 1986; Morgan y Goldston, 1987), 
constatando beneficios sobre la salud mental que contribuirán a la mejora de sentimientos de bienestar, estado de meditación o distracción de los estímulos causantes de la ansiedad (Folkins y Sime, 1981, citado en Márquez, 1995).

Estudios realizados con personas adultas de mediana edad corroboran el nexo positivo establecido entre la práctica física y la aparición de estados emocionales positivos, reduciendo la ansiedad o el estrés y contribuyendo a un funcionamiento cognitivo más satisfactorio (Etnier et al., 1997; Conroy et al. 2007). Sin embargo, no queda tan clara esa relación de ejercicio físico y estados de ánimo positivos con las personas mayores, ya que no hay una importante asociación entre las motivaciones, grado de satisfacción y estados de ánimo derivados de la práctica de ejercicio físico (Keller y Woolley, 1991). Este sector poblacional es susceptible a los estados de ánimo negativos o bajos, no siendo esto una vinculación generalizada o afirmativa. Esto es el reflejo de un estudio que se realizó con 319 coreanos ancianos, mayores de 60 años, a los cuales se les aplicó el Profile of Mood States (POMS) en su versión original. Los resultados mostraron que las personas mayores coreanas percibían buen estado de ánimo por lo general, pero cuanto mayor eran sus edades e incluso si pertenecían al género femenino, menos positivos eran los estados de ánimo que presentaban (Shin y Colling, 2000).

Las dimensiones del estado de ánimo pueden ser negativas o positivas. Para su evaluación, se debe obtener un determinado índice general de alteración del estado de ánimo y siete medidas parciales como son los estados de Tensión (tensión muscular, intranquilidad y ansiedad), Depresión (sensación de infelicidad), Cólera (ira y antipatía hacia los demás), Vigor (estado eufórico y con mucha energía), Fatiga (estado de poca energía), Confusión (indeciso) y Amistad (social) (Andrade, Arce y Seaone, 2000). Estas escalas conforman el POMS, compuesta por 65 ítems (McNair, Lorr y Droppleman, 1971) mediante un formulario tipo Likert de 5 alternativas que van desde el 0 (nada) a 4 (muchísimo). Posteriormente se han empleado otras versiones, todas ellas derivadas del original, pero reducidas o justificando la eliminación de algún ítem (Watson y Clark, 1997; Andrade, Arce y Seaone, 2008). Una muestra de aplicación del POMS reducido, es la compuesta por Balaguer, Fuentes, Meliá, García y Pérez (1993), que consta de 29 ítems que describen a su vez cinco estados de ánimo diferentes como son la tristeza, el vigor, la cólera, la tensión y fatiga cuyos valores de alfa de Cronbach en la versión original oscilatorios entre 0.70 y 0.83 . Otro POMS reducido se llevó a cabo en un trabajo realizado en Alemania, compuesta por 35 ítems, con unos valores alfa de Cronbach de 0.89 a 0.95 y formada por cinco de estados de ánimo distintos del estudio anterior como son depresión, tensión, fatiga, vigor y cólera (Albani et al., 2005).

Teniendo en cuenta lo expuesto anteriormente y sentando las bases de nuestro estudio, nos proponemos a examinar los vínculos que se establecen entre la práctica de actividad física aeróbica, estados de ánimo y variables demográficas en un contexto geográfico concreto. Esto facilitará la concreción del objetivo de este trabajo, el cual es analizar la repercusión la practica de actividad física aeróbica en de diferentes variables (edad, enfermedad, antigüedad y motivos en el programa de actividad física) y su influencia sobre cuatro estados de ánimo concretos (tensión, vigor, fatiga y amistad).

\section{Método}

\subsection{Diseño}

Para la presente investigación, se ha utilizado un diseño no experimental, descriptivo, seccional (Sierra, 2001, p.14).

\subsection{Muestra}

Como universo de estudio, se ha establecido a los participantes en el programa de Actividad Física para personas Mayores de la Diputación de Málaga. Concretamente nos situamos en los sujetos pertenecientes a tres poblaciones de menos de 3.000 habitantes de una misma comarca, ubicadas geográficamente en la Sierra de las Nieves. Se corresponde a cuatro grupos de mujeres, que no presentan ningún interés especial a la hora de realizar actividades físico-deportivas aeróbicas, mediante aeróbic y/o diferentes coreografías, en salas habilitadas para la realización de la misma.

Este programa lleva siete años en funcionamiento y consta de una asiduidad de dos sesiones semanales. Estas sesiones se realizan a primeras horas de la mañana, respetando el ritmo circadiano individual y con una duración de 45 a 50 minutos aproximadamente.

Nuestra muestra se centra concretamente en el género femenino, de manera que, dentro de una población finita y para un error muestral de $\pm 2 \%$, corresponde la muestra a un total de 63 sujetos de investigación. Estas mujeres, presentan edades de 60 a 79 años, $(M=65,98$; DT= 5,28 ), las cuales hemos divididos en dos rangos de edad de diez años.

En la tabla 1 se muestra la distribución de la muestra en función de las preguntas realizadas en el cuestionario sociodemográfico, como son la edad, la enfermedad limitante de práctica física, la antigüedad en el programa de condición física y los motivos de práctica.

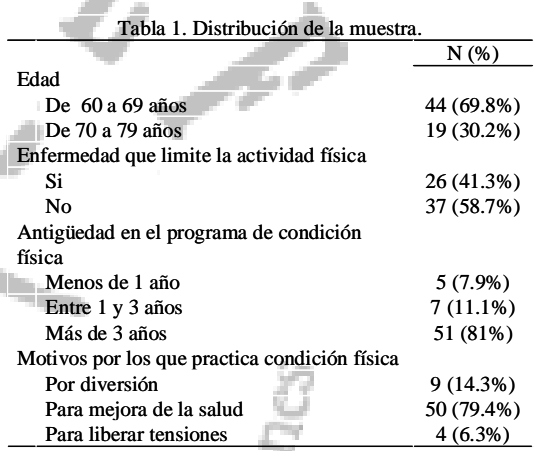

\subsection{Instrumentos}

Se ha utilizado como técnica de investigación la encuesta, destacando como instrumento el cuestionario. Siguiendo a Sierra (2001, p.306), el cuestionario que hemos utilizado junto con el POMS, es el cuestionario simple, siendo uno de los instrumentos de la técnica de investigación por encuestas (Colás y Buendía, 1994; Manzano, Rojas y Fernández, 1996; Arias y Fernández, 1998); considerado como el instrumento básico de recogida de información de la investigación social mediante encuestas (García Ferrando, 2000).

El cuestionario sociodemográfico se ha estandarizado a través del Coeficiente de correlación de Spearman, que nos informa de la estabilidad del cuestionario. Concretamente hemos realizado las correlaciones de cada ítem del test con su correspondiente en el retest, donde un 93\% de las correlaciones resultaron positivas o significativas, lo que es indicativo de la estabilidad del mismo. De igual forma, se ha hallado el coeficiente alfa de Cronbach en el test y retest, obteniéndose valores por encima de 0.72 , lo cual indica un alto grado de fiabilidad. Posteriormente, hemos solicitado la revisión de dicho instrumento a varios expertos en esta metodología de investigación. Este cuestionario se compone de cuatro preguntas, siendo la primera de ellas de tipo abierta, mientras que las otras tres son de tipo categorizadas (Sierra, 2001). Las tres últimas variables han sido útiles para obtener información a cerca de la antigüedad de las participantes en el Programa de Actividades Físico-Deportivas, la presencia de alguna enfermedad que les limite la práctica de actividad física y el motivo de la asistencia a dicha actividad. Se aclaró a las participantes que se entendía por enfermedad limitante de práctica física cualquier dolencia o enfermedad que les impidiese desarrollar de manera normal los diferentes contenidos trabajados en el transcurso de las dos sesiones.

Para medir los cambios en los estados de ánimo se ha aplicado el POMS (McNair, Lorr y Droppleman, 1971) en su adaptación al castellano por Andrade, Arce y Seaone (2000). Se ha tomado cuatro estados de ánimo que se identifican con dos estados emocionales positivos (amistad y vigor) y dos negativos (tensión y fatiga), con unos valores alfa de Cronbach que oscilan entre 0.60 y 0.72 y compuestos por un total de 30 adjetivos (ítems) (Tabla 2). Cada uno de ellos, han sido evaluados por cada participante mediante una escala Likert de 0 (nada) hasta 4 (muchísimo) y tras la formulación de la pregunta: 
Tabla 2: Composición del cuestionario utilizado, con 30 ítems (Arce, Andrade y Seaone, 2000).

\begin{tabular}{|c|c|}
\hline Estado & Ítems \\
\hline \multirow{8}{*}{$\begin{array}{l}\text { Tensión } \\
\text { (8 Ítems) }\end{array}$} & 2. Tenso \\
\hline & 10. Agitado \\
\hline & 16. Con los nervios de punta \\
\hline & 21. Relajado \\
\hline & 24. Intranquilo \\
\hline & 25. Inquieto \\
\hline & 32. Nervioso \\
\hline & 39. Ansioso \\
\hline \multirow{8}{*}{$\begin{array}{l}\text { Vigor } \\
\text { (8 İtems) }\end{array}$} & 7. Animado \\
\hline & 15. Activo \\
\hline & 19. Enérgico \\
\hline & 36. Alegre \\
\hline & 49. Alerta \\
\hline & 54. Lleno de energía \\
\hline & 58. Libre de preocupaciones \\
\hline & 61. Vigoroso \\
\hline \multirow{7}{*}{$\begin{array}{l}\text { Fatiga } \\
\text { (7 Ítems) }\end{array}$} & 4. Rendido \\
\hline & 11. Desatento \\
\hline & 27. Fatigado \\
\hline & 38. Exhausto \\
\hline & 44. Débil \\
\hline & 47. Cansado \\
\hline & 63. Agotado \\
\hline \multirow{7}{*}{$\begin{array}{l}\text { Amistad } \\
\text { (7 Ítems) }\end{array}$} & 1. Amistoso \\
\hline & 6. Sensato \\
\hline & 13. Considerado (con los demás) \\
\hline & 23. Comprensivo \\
\hline & 28. Servicial \\
\hline & 41. Amable \\
\hline & 53. Confiado \\
\hline
\end{tabular}

«icómo te has sentido tras la realización de las dos últimas clases de aeróbic?».

2.4 Procedimiento

El estudio se llevó a cabo siguiendo las normas d e on tológ i c a s reconocidas por la Declaración de Helsinki (revisión de Hong-Kong, septiembre de 1989) y de acuerdo con las recomendaciones de Buena Práctica Clínica de la CEE (documento 111/ 3976/88 de julio de 1990) y la normativa legal vigente española que regula la investigación.

Primeramente, se realizó actividad física aeróbica (aeróbic) en el

transcurso de 16 semanas, con la frecuencia y duración especificadas. Ambos cuestionarios fueron presentados en un mes no atípico como puede ser Mayo, Agosto o Diciembre, con el fin de que ningún otro factor pudiese afectar emocional ni afectivamente a los resultados futuros de esta investigación. Al finalizar la segunda sesión de la semana, se les presentó a las participantes dos instrumentos mediante dos hojasdocumentos, los cuales fueron leídos en una primera fase para destacar posibles incomprensiones. Posteriormente, se procedió a realizar el cuestionario sociodemográfico con las variables consideradas a continuación:

-Edad: Los grupos que se crearon se estratificaban en dos franjas de edad que oscilaban entre los 60 a 69 años y 70 a 79 años. Tanto uno como otro grupo, se establecieron a partir de las frecuencias obtenidas de la muestra total de todas las mujeres que colaboraron en el proceso de obtención de datos.

-Enfermedad que limite la práctica de actividad física: $\mathrm{Si}$ (problemas que limitasen la movilidad de cadera, fracturas importantes o cardiopatías graves como anginas, soplos o infartos) y no (incluyendo dolores musculares -óseos ocasionales y alguna patología relacionada con la espalda pero con leve incidencia).

-Antigüedad en el programa: menos de 1 año (dándose el caso de alguna mujer que llevase un solo mes), entre 1 y 3 años, más de tres años (incluso participantes que comenzaron con el nacimiento del programa hace siete años).

-Motivos de práctica de actividad física: por diversión, mejora de salud (física o mental) y para liberar tensiones (con fuertes matices de función catártica y hedonista).

Las variables dependientes se presentan en una segunda hoja con una lista vertical de los 30 adjetivos del POMS y al estado de ánimo al que se correspondía. A la izquierda de cada ítems/adjetivo se disponían cinco recuadros para facilitar su realización.

Todo el procedimiento llevado a cabo fue individual y sin ningún límite de tiempo, con la presencia de un componente de este estudio para resolver cualquier problema y hacer que se siguiesen las pautas establecidas.

Los datos recogidos han sido tratados con el programa SPSS 15.0 para windows. Para las variables edad, enfermedades, antigiedad, motivos de práctica física y estados de ánimo, se ha calculado los estadísticos descriptivos media y desviación típica. La significatividad en la diferencia de los grupos de edad y si se tiene enfermedad o no se ha establecido mediante la $t$ de Estudent y el ANOVA de un factor para la antigüedad y motivos de práctica, también respecto a los estados de ánimo.

\section{Resultados}

Para los resultados obtenidos en cuanto niveles de tensión, vigor, fatiga y amistad en función de la edad, las mujeres que se sitúan en la franja de más edad (70 a 79 años) presentan más niveles de tensión, a la vez que de vigor y amistad. No es así con el estado de ánimo de fatiga, siendo menor que las mujeres de menor edad. En cuanto la relación de los cuatro estados de ánimo con los motivos por los que se practica condición física, la mejora de la salud es la que presenta mayores niveles respecto a tensión, fatiga y amistad, no siendo así con el estado de vigor. En ambas variables independientes (edad y motivos de práctica física) no se encuentran diferencias significativas. Si se observaron tales diferencias en el nivel de tensión entre las participantes que si tenían una enfermedad que le limitaba la práctica física y las que no $(\mathrm{t} 61=2,35$, p< 0.05 ), $\mathrm{y}$ entre los diferentes años de antigüedad que llevaban en el programa de condición física $(\mathrm{F}(2,60)=3,15, \mathrm{p}<0.05)$.

La distribución muestral por porcentajes refleja la gran participación de mujeres más próximas a la edad adulta que a la vejez, correspondiéndose un $69.8 \%$ para la estratificación poblacional de 60 a 69 años y un $30.2 \%$ para las que se encuentran entre los 70 y 79 años. También se ha visto que la enfermedad no es una limitación para la práctica regularde actividad física, ya que el $41.3 \%$ de la mujeres presentan algún tipo de enfermedad respecto al $58.7 \%$ que no padecen. El resultado más destacado es la antigüedad que llevan en el programa (Figura 1) y su consecuente práctica regular de ejercicio físico. Un $81 \%$ lleva más de tres años en el programa, mientras que un $11.1 \%$ lleva entre tres y un año, y un $7.9 \%$ lleva menos de uno. Similar es el motivo de práctica física (Figura 2), siendo notoria la cifra de $79.8 \%$ para las practicantes que asisten para mejorar su salud, frente el $14 \%$ que lo hacen por diversión y un $6.3 \%$ para liberar tensiones.

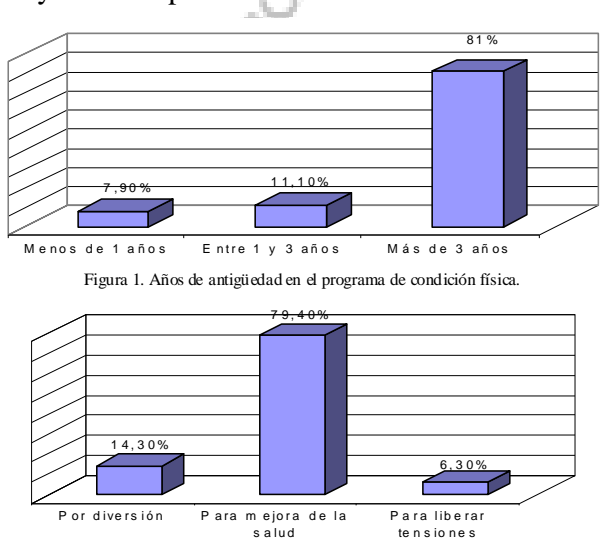

Figura 2. Motivos por el que las mujeres practica actividad física.

En la tabla 3 se puede apreciar los estados de tensión, vigor, fatiga y amistad teniendo en cuenta la edad, enfermedad limitante de práctica física, antigüedad en el programa y motivos por los que se acude al mismo.

\section{Discusión y conclusiones}

Con los datos obtenidos a través del presente estudio, nos proponemos demostrar nuestro objetivo inicial, el cual es analizar los efectos de las variables edad, enfermedad, antigüedad y motivos de práctica, en un trabajo físico aeróbico de mujeres entre 60 y 79 años. Todo ello sobre cuatros estados de ánimo, dos negativos (tensión y fatiga) y dos positivos (vigor y amistad) del POMS, en su traducción al castellano (Balaguer et al., 1993; Balaguer, Fuentes, Meliá, García y Pons, 1994; Andrade, Arce y Seaone, 2000).

La práctica de actividad física de manera regular, es un factor que determina la calidad de vida produciendo entre otros, beneficios psicológicos (Paffenbarger y Powell, 1985; Blasco, Capdevilla y Cruz, 1994). Cabe decir, que la falta de ejercicio físico determina claramente la aparición de enfermedades, sobre todo a cierta edad como la que nos ocupa (Gómez, Santandreu y Egea, 1995). 


\begin{tabular}{|c|c|c|c|c|c|}
\hline & & Tensión & Vigor & Fatiga & Amistad \\
\hline & & $\begin{array}{c}\mathrm{M} \\
\text { (DT) }\end{array}$ & $\begin{array}{c}\mathrm{M} \\
\text { (DT) }\end{array}$ & $\begin{array}{c}\mathrm{M} \\
\text { (DT) }\end{array}$ & $\begin{array}{c}\mathrm{M} \\
\text { (DT) }\end{array}$ \\
\hline \multicolumn{6}{|l|}{ Edad } \\
\hline & \multirow[t]{2}{*}{ De 60 a 69 años } & 3.81 & 26.40 & 4.68 & 22.52 \\
\hline & & $(2.95)$ & 3.32) & (3.39) & (4.30) \\
\hline & \multirow[t]{2}{*}{ De 70 a 79 años } & $\begin{array}{c}4.42 \\
(3.59)\end{array}$ & $\begin{array}{l}28.05 \\
(2.91)\end{array}$ & $\begin{array}{c}4.52 \\
(3.74)\end{array}$ & $\begin{array}{l}23.00 \\
(3.80)\end{array}$ \\
\hline & & -.69 & -1.86 & .16 & -.41 \\
\hline \multicolumn{6}{|c|}{ Enfermedad que limite la actividad física } \\
\hline & \multirow[t]{2}{*}{$\mathrm{Si}$} & 5.07 & 26.59 & 4.65 & 22.88 \\
\hline & & $(3.63)$ & (3.57) & (3.27) & (3.62) \\
\hline & \multirow[t]{2}{*}{ No } & 3.24 & 27.34 & 4.62 & 22.51 \\
\hline & & $(2.54)$ & (2.78) & (3.65) & $(4.50)$ \\
\hline & $t$ & $2.35^{*}$ & .89 & .03 & .34 \\
\hline \multicolumn{6}{|c|}{ Antigüedad en el programa de condición física } \\
\hline & \multirow[t]{2}{*}{ Menos de 1 año } & 4.41 & 24.14 & 3.00 & 21.80 \\
\hline & & $(3.31)$ & (4.63) & (2.64) & (3.11) \\
\hline & \multirow[t]{2}{*}{ Entre 1 y 3 años } & 2.14 & 27.17 & 3.14 & 22.14 \\
\hline & & (.89) & (3.01) & (1.86) & (3.48) \\
\hline & \multirow[t]{2}{*}{ Más de 3 años } & 2.40 & 28.0 & 5.0 & 22.82 \\
\hline & & $(1.94)$ & (2.12) & (3.64) & (4.33) \\
\hline & $F$ & 2.40 & $3.15^{*}$ & 1.5 & 19 \\
\hline \multicolumn{6}{|c|}{ Motivos por los que practica condición física } \\
\hline & Por diversión & 3.11 & 27.11 & 4.55 & 22.22 \\
\hline & \multirow{2}{*}{ Por mejora de la salud } & 4.3 & 26.72 & 4.74 & $\begin{array}{l}(3.90) \\
22.76\end{array}$ \\
\hline & & $(3.32)$ & $(3.41)$ & (3.48) & $(4.26)$ \\
\hline & \multirow[t]{2}{*}{ Para liberar tensiones } & 2.25 & 28.75 & 3.5 & 22.5 \\
\hline & & $(1.89)$ & $(2.87)$ & (3.0) & (3.69) \\
\hline & $F$ & 1.21 & .72 & .23 & .06 \\
\hline
\end{tabular}

como depresión, ansiedad y trastornos de pánico (Babyak et al., 2000; Paluska y Schwenk, 2000). El presente trabajo tiene en cuenta las conclusiones de dichos estudios, no siendo las mismas características tanto en frecuencia y duración de las sesiones, ni tampoco provocando sensaciones tan similares en las mujeres mayores. Todo ello se refleja en la variable que aborda los motivos de práctica de actividad física por parte de las integrantes del programa, siendo uno de ellos para liberar tensiones con un $6.3 \%$ del total y por diversión un $14.3 \%$. Los dos motivos con menos porcentajes de los tres existentes. La variable que falta es la que representa el mayor porcentaje de mujeres (79.4\%), que acuden al programa para una mejora de la salud aunque no se encuentre asociación con ningún estado de ánimo en concreto.

En definitiva, de todo este proceso selectivo, tanto de obtención de datos como análisis de resultados, se pueden extraer diversas conclusiones. Una de ellas es que la muestra aquí estudiada y perteneciente al programa de Actividad Física para mayores de la Diputación de Málaga, en su mayoría ( $81 \%$ del total), llevan más de tres años inscritas a dicha actividad, con lo que la antiguiedad y práctica asidua influye en el estado de vigor. Muchas de ellas llegando a inscribirse el primer día que el programa se puso en marcha hace ya siete años. Con ello, queremos destacar un aspecto muy importante en la vida diaria de estas mujeres y es la dependencia que presentan con el deporte y concretamente con este programa, el cual se sienten bastante identificadas. Al hablar de estados de ánimo, la tensión muscular esquelética post ejercicio físico es más notoria en la variable que explicita la existencia o no de enfermedades más que ninguna otra, ya que la diferencia se acentúa más. Resaltar que los mayores índices de vigor y amistad lo presentan las mujeres más mayores (70 a 79 años), coincidiendo con las aportaciones de Janisse, Nedd, Escamilla y Nies (2004), McLafferty, Wetzstein y Hunter, (2004) quienes afirman que el ejercicio físico contribuye a aumentar el bienestar psicológico, aumentando el estado de ánimo.

También este grupo de edad de 70 a 79 años, presenta menos fatiga que las más jóvenes. Esto puede ser debido a que a la hora de realizar ejercicio físico, la intensidad no sea tan elevada ya sea por limitaciones fisiológicas o simplemente por que su nivel de percepción de la actividad es menor que las que tienen menos edad. Los motivos de realización de actividad física son tres, concretamente la mejora de la salud es el mayoritario y por el cual las mujeres acuden regularmente a realizar actividad física. Este es sensiblemente mayor en todos los estados de ánimo (excepto vigor) respecto a los otros motivos; para liberar tensiones y por amistad.

En investigaciones futuras, se podría contemplar el estudio de los mismos o de diferentes estados de ánimo. Se podría realizar el POMS tras la participación en diferentes actividades, como no aeróbicas o anaeróbicas, en diferentes medios como el acuático, natural o incluso en diferentes superficies como parquet, arena de playa, terreno duro, asfalto o en la propia naturaleza. También se puede extender a diferentes poblaciones (rurales, poblaciones de menos de 10.000 habitantes, ciudades, centros educativos y deportivos o centros de mayores). Con diferente género (mujeres y hombres) tanto juntos como por separados y con otras variables a tratar como el nivel de estudios o práctica física fuera del programa de condición física al que hace referencia este trabajo.

\section{Referencias}

Abbott, A. (2004). Ageing: Growing old gracefully. Nature, 428, 116-8.

Acuña, L. y Bruner, C.A. (2002). El efecto de las personas sobre la salud. Revista Mexicana de Psicología, 19(2), 115-124.

Albani, C., Blaser, G., Geyer, M., Schmutzer, G., Brahler, E., Bailer, H. y Grulke, N. (2005). The German short version of «Profile of mood states» (POMS): Psychometric evaluation in a representative simple. Psychotherapie Psychosomatik Medizinische Psychologie, 55, 324330.

Andrade, E., Arce, C., Armental, J., Rodríguez, M. y De Francisco, C. (2008). Indicadores del estado de ánimo en deportistas adolescentes según el modelo multidimensional del POMS. Psicothema, 20 (4), 630-635.

Andrade, E., Arce, C. y Seaone, G.P. (2002). Adaptación al español del cuestionario «Perfil de los Estados de Ánimo» en una muestra de deportistas. Psicothema, 14 (4), 708-713.

(Cassidy et al., 2004). Se ha demostrado que el ejercicio aeróbico, por ejemplo realizado durante 30 minutos al menos tres días por semana, tiene un potente efecto terapéutico sobre algunas enfermedades mentales, 
Andrade, E., Arce, C. y Seaone, G.P. (2000). Problemas semánticos en la adaptación del POMS al castellano. Psicothema, 12 (2), 47-51.

Babyak, M., Blumenthal, J-A, Herman, S., Khatri, P., Doraiswamy, M., Moore, K., (2000). Exercise treatment for major depression: maintenance of therapeutic benefit at 10 months. Psychosom Medicine, 62, 633-8.

Balaguer, I., Fuentes, I., Meliá, J.L., García, M.L., y Pérez, G. (1993). El Perfil de los Estados de Ánimo (POMS): baremo para estudiantes valencianos y su aplicación en el contexto deportivo. Revista de Psicología del Deporte, 4, 39-52.

Balaguer, I., Fuentes, I., Meliá, J.L., García, J.L., y Pons, D. (1994). Adaptación del Perfil de Estados de Ánimo (POMS) a una muestra de estudiantes valencianos. Actas del IV Congreso de Evaluación Psicológica. Santiago de Compostela.

Benaziza, H. (1998). Creación de asociaciones: una clave para promover la actividad física para la salud. Actas del VII Congreso Mundial de Deporte para Todos. El deporte para todos y los retos educativos a nivel mundial. Barcelona.

Biddle, S. (1993). Psychological benefits of exercise and physical activity. Revista de Psicología del Deporte, 4, 99-107.

Biddle, S.J.H., Fox, K.R. y Boutcher, S.H. (2000). Physical activity and psychological wellbeing, Londres: Routledge.

Blasco, T., Capdevila, LL. y Cruz, J. (1994). Relaciones entre actividad física y salud. Anuario de psicología, 6 (12), 19-24.

Cassilhas, C., Viana, V., Grassmann, V., Santos, R.T., Santos, F., Tufik, S y Melo, T. (2007). The impact of resistance exercise on the cognitive function of the elderly. Medecine and Science in sports and exercise, 39 (8), 1401-1407.

Cassidy, K., Kotynia-English, R., Acres, J., Flicker, L., Lautenschlager, N.T. y Almeida, O.P. (2004). Association between lifestyle factors and mental health measures among community-dwelling older women. Australian and New Zealand Journal of Psychiatry, 38, 940-947.

Chachamovich, E., Fleck, M., Laidlaw, K. y Power, M. (2008). Impact of Major Depression and Subsyndromal Symptoms on Quality of Life and Attitudes Toward Aging in an International Sample of Older Adults. Gerontologist, 48, 593-602.

Conroy, M., Simkin-Silverman, L., Pettee, K., Hess, R., Kuller, L. y Kriska, A. (2007). Lapses and psychosocial factors related to physical activity in early pstmenopause. Medicine and Science in Sports and Exercise, 39(10), 1858-1866.

Corrales, E., Tardón, A. y Cueto, A. (2000). Estado funcional y calidad de vida en mayores de setenta años. Psicothema, 12 (2), 171-175.

Daniels, R.; Van Rossum, E.; De Witte, L.; Kempen, G.I. y Van den Heuvel, W. (2008). Interventions to prevent disability in frail community-dwelling elderly: a systematic review. BMC Health services research, 30, 278-286.

Danner, R. y Edward, D. (1992). Life is movement: Exercise for the older adult. Activities, Adaptation and Aging, 17 (2), 15-26.

De Gracia, M. y Marcó, M. (2000). Efectos psicológicos de la actividad física en personas mayores. Psicothema, 12 (2), 285-292.

Dishman, R.K. (1986). Mental health. Physical activity and well-being. Reston: American Alliance of Health, Physical Education, Recreation and Dance.

Dishman, R.K. (1994). Advances in Exercise Adherence. Illinois: Human Kinetics.

Dorn, J., Naughton, J., Imamura, D. y Trevisan, M. (1999) Results of a multicenter randomized clinical trial of exercise and long-term survival in myocardial infarction patients: the National Exercise and Heart Disease Project (NEHDP). Circulation, 100, 1764-9.

Endres, M., Gertz, K., Lindauer, U., Katchanov, J., Schultze, J. y Schröck, H., (2003) Mechanisms of stroke protection by physical activity. Annals of Neurology, 54, 582-90.

Erikssen, G. (2991). Physical fitness and changes in mortality: the survival of the fittest. Journal of Sports Medicine, 31, 571-6.

Etnier, J.L., Salazar, W., Landers, D.M., Petruzzello, S.J., Han, M., y Nowell, P. (1997). The Influence of Physical Fitness and Exercise Upon cognitive Functioning: A Meta Analysis. Journal of Sport and Exercise Psychology, 19, 249-277.

Folkins, C. H. y Sime, W. E. (1981). Physical fitness training and mental health. American Psychologist, 36, 373-389.

García Ferrando, M. (2005), Posmodernidad y Deporte: Entre la Individualiuzación y la Masificación. Encuesta hábitos deportivos de los españoles, Madrid, CIS/Siglo XXI.

Gulati, M., Pandey, D.K., Arnsdorf, M.F., Lauderdale, D.S, Thisted, R.A y Wicklund RH (2003). Exercise capacity and the risk of death in women: the St James Women Take Heart Project. Circulation, 108, 1554-9.

Janisse, H., Nedd, D., Escamilla, S. y Nies, M. (2004). Physical activity, social support, and family structure ad determinants of mood amons European-American and African-American women. Women Health, 39 (1), 101-116.

Jiménez, M., Martínez, P., Miró. E. y Sánchez. A. (2008). Bienestar psicológicos y hábitos saludables: ¿Están asociados a la práctica de ejercicio físico?. Internacional Journal of Clinical and Health Psichology, 8 (1), 185-202.

Keller, M.J. y Woolley, S.M. (1991). Designing exercise programs with older adults: Theory and practice. Activities, Adaptation and Aging, 2, 1-17.

Lee, C.D., Folsom, A.R. y Blair, S.N. (2003). Physical activity and stroke risk. Stroke, 34, 2475-81.

Maroulakis, E., Zervas I (1993). Effects of aerobic exercise on mood of adult women. Perceptual and motor skill, 76, 795-801.

Márquez, S. (1995). Beneficios psicológicos de la actividad física. Revista de psicología general y aplicada, 48 (1), 185-206.

McAuley, E., Mihalko, S.L. y Bane, S.M. (1997). Exercise and selfesteem in middle-aged adults: Multidimensional relationships and physical fitness and self-efficacy influences. Journal of Behavioural Medicine, 20, 67-83.

McCann, L. y Holmes, D. (1984). Influence of aerobic exercises on depression. Journal of personality and social psychology, 46 (5), 1142-1147.

McLafferty, C., Wetzstein, C. y Hunter, G. (2004). Resistance training is associated with improved mood in healthy older adults. Perceptual and Motor Skills, 93 (3), 947-957.

McNair, D.M., Lorr, M. y Droppleman, L.F. (1971). Manual for the Profile of Mood States. San Diego, CA: Educational and Industrial Testing Service.

Moreno González, A. (2005). Incidencia de la Actividad Física en el adulto mayor. Revista Internacional de Medicina y Ciencias de la Actividad Física y el Deporte vol. 5 (19) 222-237

Morales, R. y Coto, V.E. (2004). Efecto agudo en el estado anímico del adulto mayor al realizar ejercicios con pesas a diferentes intensidades, para aplicarla como tratamiento en dicha población. Intercedes: Revista de las Sedes Regionales, 5 (7).

Morgan, W.P. y Goldston, S.E. (1987). Exercise and mental health. Washington: Hemisphere

Myers, J., Prakash, M., Froelicher, V., Do, D., Partington, S. y Atwood,

J.E. (2002). Exercise capacity and mortality among men referred for exercise testing. The New England of Journal Medicine, 346, 793-801.

Olmedilla Zafra, A., Ortega Toro, E. y Madrid Garrido, J. (2008). Variables sociodemográficas, ejercicio físico, ansiedad y depresión en mujeres: un estudio correlacional. Revista Internacional de Medicina y Ciencias de la Actividad Física y el Deporte vol. 8 (31), 224-243.

Paluska, S.A. y Schwenk, T.L. (2000). Physical activity and mental health: Current Concepts. Journal of Sports Medicine, 29, 167-80.

Pierce, E. y Pate, D. (1994). Mood alterations in older adults following acute exercise. Perceptual and motor skills, 79 (1), 191-194.

Rosenfeld, O. y Tenembaum, G. (1992). Physical fitness in the industrial environment: perceived physical well-being benefits. International Journal of Sport Psychology, 23, 227-242.

Segunda Asamblea Mundial sobre el Envejecimiento (2000). Madrid: ONU.

Shin, Y., y Colling, K.B. (2000). Cultural verification and application of the Profile of Mood States (POMS) with Korean elders. Western Journal of Nursing Research, 22, 68-83.

Sierra Bravo, R. (2001). Técnicas de investigación social. Teoría y ejercicios. Madrid: Ed. Paraninfo Thomson Learning.

Schwerdtfeger, A., Eberhardt, R., y Chmitorz, A. (2008). Is there a correlation between everyday-life physical activity and psychological well-being? A methodological study using ambulatory monitoring. Zeitschrift fur gesundheitspsychologie, 16 (1), 2-11

Van Heuvelen, M.J.G., Hochstenbach, J.B.H., Brouver, W.H., De Greef, M.H.G. y Scherder, E. (2006). Psychological and physical activity training for olders persons: who does not attend?. Gerontology, 52, 366-375.

Varo, J.J., Martínez, J.A. y Martínez-González, M.A. (2003). Beneficios de la actividad fisica y riesgos del sedentarismo. Medicina Clinica (Barc), 121, 665-72.

Watson, D., y Clark, L.A. (1997). Measurement and mismeasurement of mood: Recurrent and emergent issues. Journal of Personality Assessment, 68, 267-296. 